\title{
Lateral Supraorbital Approach for the Surgical Clipping of Ruptured Tiny Anterior Circulation Aneurysms
}

\author{
Tao Jiang ${ }^{1,2}$, Lili Yan ${ }^{3}$, Mingyu Chen ${ }^{3}$, Junfei $\mathrm{Cao}^{3}$, Yanjie Shen ${ }^{3}$ and Zhenhua Ren ${ }^{3}$ \\ ${ }^{1}$ Department of Neurosurgery, The Fourth Affiliated Hospital of Anhui Medical University, Hefei, Anhui, China \\ ${ }^{2}$ Department of Neurosurgery, The Second Affiliated Hospital of Anhui Medical University, Hefei 230601, Anhui, China \\ ${ }^{3}$ Department of Anatomy, School of Basic Medicine, Anhui Medical University, Hefei, Anhui, China
}

\begin{abstract}
Objective: To evaluate the efficacy and usefulness of clipping procedure by lateral supraorbital approach for tiny anterior circulation aneurysms.

Study Design: An observational study.

Place and Duration of Study: Department of Neurosurgery, The Second Hospital of Anhui Medical University, Hefei, Anhui Province, China, from January 2013 to March 2018.

Methodology: Thirty-eight patients who had suffered aneurysm rupture, resulting in subarachnoid hemorrhage, were selected. Fourty-four very small aneurysms with a maximum diameter of $\leq 3 \mathrm{~mm}$ that presented different configurations, were identified using digital subtraction angiography (DSA). Clinical data of patients, including clinical information, microsurgery procedure, procedural complications, clinical outcomes, and follow-up were analysed.

Results: All ruptured aneurysms involved the anterior circulation, located in middle cerebral artery (19/38), anterior communicating artery (10/38), posterior communicating artery (6/38), anterior choroidal artery (2/38), and ophthalmic artery (1/38). Complete occlusions were achieved in all of the lesions by surgical clipping via the lateral supraorbital approach. There were no intra-procedural ruptures or procedure-related vasospasm, epilepsy, hydrocephalus. Glasgow outcome scale (GOS) scores showed that 34 patient $(86.84 \%)$ had good outcomes (GOS 4-5) at the time of discharge. There was no residual neck or recurrence following computed tomography angiography (CTA) and DSA at follow-up in 20 cases.

Conclusion: Surgical clipping via lateral supraorbital approach presented a low rate of adverse events related to the procedure, and was safe and effective in ruptured tiny aneurysms.
\end{abstract}

Key Words: Tiny aneurysm; Surgical clipping; Clinical outcome; Lateral supraorbital approach.

How to cite this article: Jiang T, Yan L, Chen M, Cao J, Shen Y, Ren Z. Lateral Supraorbital Approach for the Surgical Clipping of Ruptured Tiny Anterior Circulation Aneurysms. J Coll Physicians Surg Pak 2020; 30(05):523-526. DOI: https://doi.org/10.29271/jcpsp.2020.05.523.

\section{INTRODUCTION}

Surgical clipping is a traditional method to repair aneurysmal dilation, introduced by Walter Dandy of Johns Hopkins Hospital in $1937 .{ }^{1,}$

${ }^{2}$ With advancement of endovascular techniques, endovascular management of ruptured intracranial aneurysms is considered a valid alternative to microsurgical clipping. ${ }^{3}$ The international subarachnoid aneurysm trial (ISAT) demonstrated the superiority of endovascular therapy. ${ }^{4}$ However, ISAT conclusion was criticised by many clinicians, and not well accepted by subsequent studies. ${ }^{5,6}$ Not all aneurysms are suitable for endovascular therapy. For tiny aneurysms ( $\leq 3 \mathrm{~mm}$ in maxium diameter), due to the characteristics of the small cavity and thin wall in which the microcatheter has to be placed, high tumor cavity pressure, as well as the increased risk of perforation related to placing coils into this tiny, fragile aneurysm $\mathrm{sac}^{7,8}$ surgical clipping is more suitable than endovascular treatment. $^{9}$

Correspondence to: Prof. Zhenhua Ren, Department of Anatomy, Anhui Medical University, 81 Meishan Road, Hefei, Anhui, 230032, China

E-mail: renzhenhua1975@163.com

Received: February 21, 2020; Revised: May 27, 2020;

Accepted: May 27, 2020

DOI: https://doi.org/10.29271/jcpsp.2020.05.523
The pterional approach described by Yasargil in 1975 became the gold standard forthetreatment of aneurysms of the anterior circulation. ${ }^{10}$ Recently, minimally invasive surgical approaches, such as the lateral supraorbital approach, have been developed. ${ }^{11,12}$ The lateral supraorbital approach may offer the following benefits: smaller skin incisions and craniotomy flap with subsequent reduced blood loss, less brain exposure, shortened operative time, reduced risk of infection, reduced postoperative pain, faster recovery and better cosmetic outcome. ${ }^{13}$ Tiny aneurysms account for almost $6 \%$ of all ruptured intracranial aneurysms. ${ }^{14}$

In this study, the aim was to determine the treatment results of clipping surgery via lateral supraorbital approach for ruptured tiny aneurysms interms offeasibility, efficacy, clinical outcomes, procedure-related risks, and safety.

\section{METHODOLOGY}

Forty-four very small aneurysms ( $\leq 3 \mathrm{~mm}$ ), with difficult configurations in 38 patients, were treated using surgical clipping via lateral supraorbital approach from January 2013 to March 2018 in The Second Affiliated Hospital, Anhui Medical University. All the clinical data used in this study were obtained from the paper and electronic medical record (EMR) of the Hospital. The study protocol was approved by the Research Ethics Commission of The Second Affiliated Hospital of Anhui Medical University. 
Gender, age, onset time, and initial manifestation of the patients were reviewed. The clinical information of the patients with tiny aneurysm, including physical sign, the location, neck and size of aneurysm, Hunt-Hess classification, were analysed. Neuroimaging findings including computed tomography angiography (CTA), digital subtraction angiography (DSA) and magnetic resonance angiography (MRA) were recorded.

Ruptured tiny aneurysms ( $\leq 3 \mathrm{~mm}$ ) were the most important inclusion criteria. The indications for surgical operation included tiny aneurysms with subarachnoid hemorrhage (SAH). History of SAH confirmed by a head computed tomography (CT) examination, which showed the hemorrhage from the aneurysms. Aneurysms larger than $3 \mathrm{~mm}$, suspected pseudoaneurysms, and aneurysms associated with brain arteriovenous malformations were excluded from the study. According to the imaging performance, surgical treatment of the patients with ruptured tiny aneurysms was mainly based on the patient preference, age, morphology of aneurysm, and the relationship between the aneurysm and its parent vessel. Final decisions regarding the treatment of patients with tiny aneurysms were discussed among the faculty members. The related risks and complications of the operation were fully communicated to the family members, and they signed the consent of the operation.

Standard procedure of surgical clipping was performed. ${ }^{11,12}$ Lateral supraorbital craniotomy was made by placing a single burr-hole 2.5 $\mathrm{cm}$ posteriorly to the keyhole point, beneath the superior temporal line. The bone flap was detached mainly by side-cutting craniotome, but basal part was drilled before lifting the flap. The free bone flap was sized from $3 \times 3$ to $4 \times 4 \mathrm{~cm}$. Dura was opened in a curved fashion with its base toward the orbital rim and the sphenoid. After opening the Sylvian fissure minimally, it was filled with water injection technique and opened proceeding towards the aneurysm site. Sylvian fissure was dissected towards midline of the opening. The arachnoid and pia maters were gradually separated from the wall to expose the aneurysm. After confirmation of the aneurysm morphology, surgical clipping of the aneurysm was performed using a mini clip. For closure, the fixation of the bone flap with two Caniofix ${ }^{\mathrm{O}, \mathrm{R}}$ was used.

The degree of occlusion was evaluated immediately post-procedure. Occlusion degree was evaluated according to the performance of postoperation CTA. According to the results of neurological examination and functional status of the patients at discharge, clinical outcome was evaluated by the Glasgow outcome scale (GOS) as follows: grade 1, deceased; grade 2, vegetative state (patient exhibits no obvious cortical function); grade 3 , severely disabled (patient depends upon others for daily support due to mental or physical disability or both), grade 4, moderately disabled (patient is independent as far as daily life is concerned. The disabilities found include varying degrees of dysphasia, hemiparesis, or ataxia, as well as intellectual and memory deficits and personality changes), and grade 5, mildly disabled or not disabled (resumption of normal activities even though there may be minor neurological or psychological deficits). One month after discharge, patients come to the centre for a follow-up visit. All patients underwent further follow-up via telephone and hospital visits at 3, 6 and 12 months following the intervention. Patients underwent a follow-up DSA at $\sim 6$ months after the intervention.

\section{RESULTS}

Thirty-eight patients underwent surgical treatment for ruptured small cerebral aneurysm. There were 14 males (36.84\%) and 24 females $(63.16 \%)$, aged 20 to 70 years. The average age was $54.90 \pm 9.38$ years. The preoperative CTA, DSA, or MRA angiography showed that there were 44 aneurysms in 38 patients. All ruptured aneurysms in 38 patients appeared in anterior circulation; $50.00 \%$ of aneurysms (19/38) were located on middle cerebral artery, $26.32 \%$ of ruptured aneurysms (10/38) on anterior communicating artery, $15.79 \%$ of aneurysms (6/38) on posterior communicating artery, $5.26 \%$ of aneurysms (2/38) on anterior choroidal artery, and one aneurysm (1/38) on ophthalmic artery. Five (13.16\%) patients had multiple aneurysms, among whom four cases (4/38) had two aneurysms, and one case (1/38) had three aneurysms. In five cases of multiple aneurysms, only one aneurysm ruptured. For an aneurysm without rupture, no surgical intervention was done as in two cases of basilar aneurysm. The average neck diameter of tiny aneurysms was $1.96 \pm 0.47 \mathrm{~mm}$.
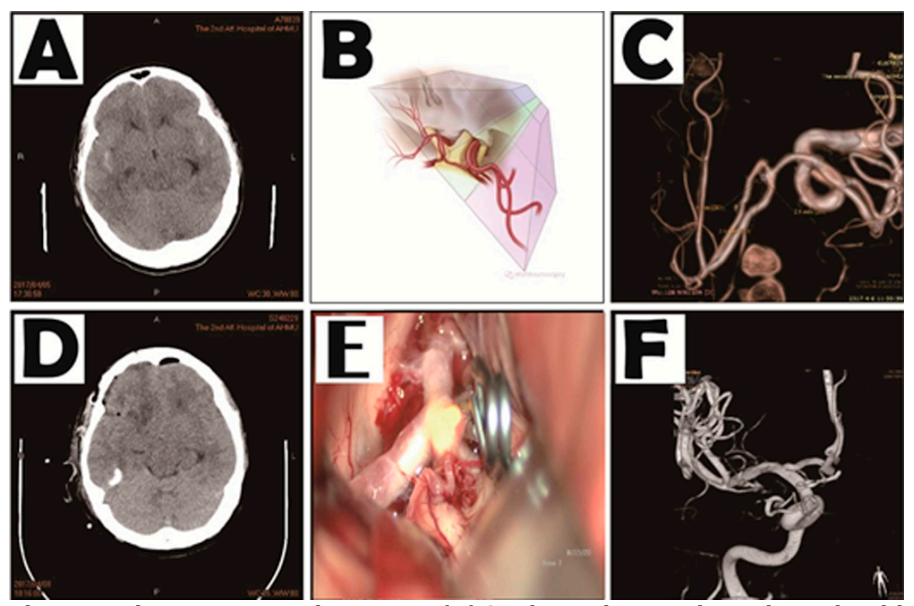

Figure 1: The representative case 1. (A) CT showed extensive subarachnoid hemorrhage at admission; (B) The schematic diagram of normal exposure range via the lateral supraorbital approach; (C) DSA angiography found a tiny aneurysm at anterior communicating artery on the second day after admission; (D) CT examination was obtained at post-operation; (E) The aneurysm had been clipped; (F) DSA angiography showed no recurrence and no residual at 3 months follow-up.
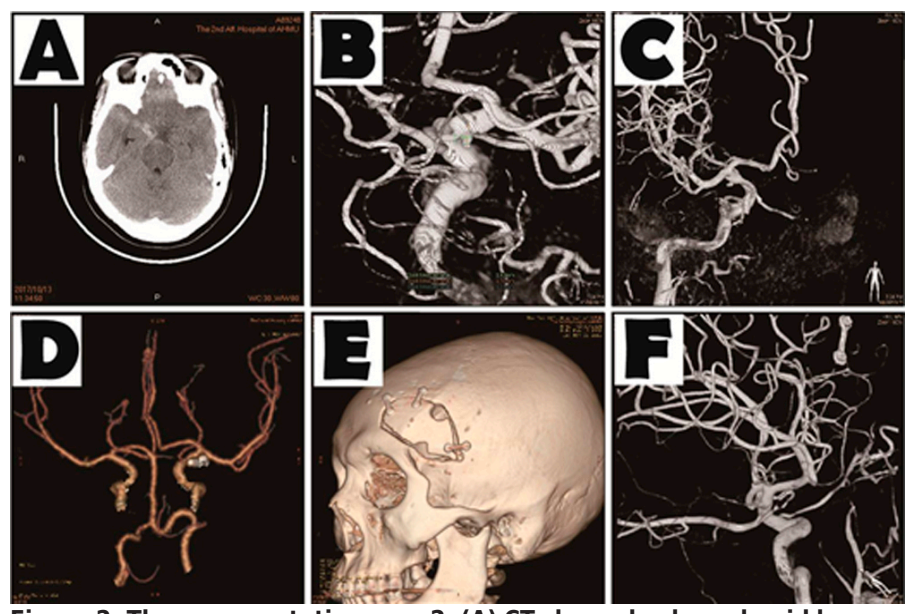

Figure 2: The representative case 2. (A) CT showed subarachnoid hemorrhage on suprasellar cistern at admission; $(B, C)$ DSA angiography found a tiny aneurysm on the location of right posterior communicating artery on the 14 days after admission; (D) After operation CTA examination was performed; (E) CTA showed the range of bone window in the operation at the discharge; (F) DSA angiography showed no recurrence and no residual at 3 months post-operation. 
All patients had suffered aneurysm-rupture, resulting in subarachnoid hemorrhage. According to Hunt-Hess classification system, $5.26 \%$ of patients $(2 / 38)$ had grade I, $52.63 \%$ of patients $(20 / 38)$ had grade II, $28.95 \%$ of patients $(11 / 38)$ had grade III, $10.53 \%$ of patients (4/38) had grade IV, and one case (1/38) had grade $\mathrm{V}$ haemorrhage, The average score was $2.53 \pm 0.86$. The initial symptoms in 35 patients (35/38) included headache $(33 / 38$, $86.84 \%)$, dizziness (8/38, 21.05\%), and vomiting (20/38, $52.63 \%)$. One case ( $1 / 38$ ) was admitted to hospital due to sudden confusion, and 2 cases (2/38) were hospitalised due to confusion and twitching of limbs. The time of onset was from 3 hours to seven days, and the average score was $25.83 \pm 62.89$ hours aneurysm location.

Aneurysm clipping was successfully achieved in all the patients (100\%). There were no surgery-related complications such as rebleeding, vasospasm, epilepsy, hydrocephalus. At the time of discharge, $86.84 \%$ of the patients (33/38) attained a favourable outcome (GOS score 4 or 5 ). Among them, 32 cases had GOS score of 5 . Only $13.16 \%$ of patients (5/38) had poor clinical outcome (GOS score 1, 2, or 3). Among the latter, one patient (1/38) died (GOS score 1 ), and 3 patient (3/38) were a vegetative state (GOS score 2 ), and 1 patient (1/38) was severely disabled (GOS score 3 ). Overall, Hunt-Hess Grades III-V were associated with poor clinical outcome. At the time of discharge, no case of residual neck or recurrence were identified by CTA.

During the follow-up, only 20 patients (52.63\%) among 38 patients received a CTA examination. Complete aneurysm occlusion was observed in all patients, and neither recanalization nor rebleeding was reported, at an average neuroradiological followupspanning 4.2 months (Figures 1 and 2 ).

\section{DISCUSSION}

Due to this arterial wall, small ruptured aneurysms may be correlated with massive bleeding and rebleeding, and it may lead to the devastating consequences. ${ }^{15}$ Consequently, effective neurosurgical clipping is usually considered for those lesions. Recently, the endovascular treatment strategy has been greatly improved, and has become an alternative strategy in the management of tiny intracranial aneurysms. ${ }^{16}$ However, management strategies for ruptured aneurysms remain controversial as the safety of endovascular coiling is still in question, and surgical treatment is technically challenging. ${ }^{16}$

The standard pterional approach is the gold standard in treating aneurysms of the anterior circulation during the following decades. ${ }^{17}$ However, this approach requires a large amount of drilling of the skull base, and removal of sphenoidanterior clinoid, possiblly resulting in many complications, including cerebrospinal fluid leakage, infection, neuromuscular injury. ${ }^{11,18}$ Recently, minimally invasive surgical approach such as the lateral supraorbital approach has been used in treating aneurysms of the anterior circulation. ${ }^{11,19,20}$ The lateral supraorbital approach was is a simpler and quicker method, and described by Hernesniemi. ${ }^{11}$ This approach may offer the following benefits, including smaller skin incisions and craniotomy flap with subsequent reduced blood loss, less brain exposure, shortened operative time, reduced risk of infection, reduced postoperative pain, and faster recovery. ${ }^{11-12,13,18}$ Moreover, it is directed more subfrontally and ante- rior than the pterional approach. ${ }^{19}$ Using the lateral supraorbital approach for tiny aneurysms, the shape and direction of the aneurysm are observable and the cerebrospinal fluid can be released, allowing removal of blood clots from the subarachnoid space, which can effectively reduce cerebral edema and cognitive impairment. ${ }^{19}$ These advantages can greatly improve the longterm prognosis of patients.

In this study, the success rate of clipping was $100 \%$, whereas $86.84 \%$ patients (33/38) attained a favorable outcome, which was sufficient to indicate the safety of the treatment. Surgical bleeding was the most important complication to consider during craniotomy. Using the lateral supraorbital approach to treat tiny aneurysms in our hospital, it was found that it was necessary to consider the association between aneurysms and the peripheral vessels and nerves based on preoperative imaging data, to better protect the peripheral vessels of the perforator. If CTA or MRI was unclear, and CTI indicated an aneurysm, an emergency DSA was required. Furthermore, it was found that placement of the head above the heartcan reduce edema of the brainand aneurysm pressure, and steering of the head would avoid the field of vision of the bony side of the skull base and the zygomatic arch. Natural brain tissue collapse allowed by CSF release in lateral orbital surgery was useful for avoiding reduction of brain tissue traction. Even if there was aneurysm on the contralateral side during operation, it can also be dealt with wholly.

Aneurysm clips were selected according to the size, thickness and angle of the aneurysm neck, and the thickness of the parent artery wall. The mini and small aneurysm clips demonstrated high application value for tiny aneurysms. Temporary clipping could be used to close the proximal artery of aneurysms which ruptured during surgery, and the double suction tube was able to cope with the bleeding. If the location of the aneurysm was difficult to reach, endoscopy was used to determine whether any residue remained or whether a further clip was required. When there is tiny blood blister like aneurysm or parent artery tear cannot be clipped, the cotton piece can be used to wrap the artery and then clip with a cross vessel clip.

\section{CONCLUSION}

Preoperative factors, including aneurysm features, surgeon preferences and economic effectiveness, should be considered prior to deciding a surgical strategy for intracranial aneurysm. Aneurysm clipping via the lateral supraorbital approach for tiny aneurysms is demonstrated to be safe, effective and wider scope ofapplication.

\section{FUNDING:}

This work was funded by the Natural Science Foundation of Anhui Higher Education Institutions of China (KJ2019A0259 and KJ2019A0940) and the Translational Medicine Research Foundation of Anhui (2017zhyx17). There were no funders involved in the study design, data analysis, manuscript preparation, or and publish the study findings.

\section{PATIENTS' CONSENT:}

Informed consents were obtained from patients to publish the data.

\section{CONFLICT OF INTEREST:}

Authors declared noconflict of interest. 


\section{AUTHORS' CONTRIBUTION:}

TJ: Collected the clinical data, and analysed data.

LY, YS, MC, JC: Performed analysis and interpretation for the data.

TJ,JC: Drafted the manuscript.

TJ, ZR: Designed and supervised the study, and modified the manuscript.

The final manuscript for publication is read and approved by all authors.

\section{REFERENCES}

1. Jo KI, Kim HR, Yeon JY, Hong SC, Kim JS. Treatment outcomes of surgical clipping for unruptured anterior circulation aneurysm-single institute experiences in the era of neurophysiologic monitoring and endovascular treatment. Neurosur rev 2015; 38:677-82.

2. Kato $Y$, Sano H, Dindorkar K, Abe M, Nagahisa S, Iwata S, et al. Treatment of unruptured intracranial aneurysms: A clinicopathological correlation. Acta neurochir 2001; 143: 681-6.

3. Gupta V, Chugh M, Jha AN, Walia BS, Vaishya S. Coil embolization of very small ( $2 \mathrm{~mm}$ or smaller) berry aneurysms: feasibility and technical issues. AJNR Am J Neuroradiol 2009; 30:308-14.

4. Molyneux A, Kerr R, Stratton I, Sandercock P, Clarke M, Shrimpton J, et al. International subarachnoid aneurysm trial (isat) of neurosurgical clipping versus endovascular coiling in 2143 patients with ruptured intracranial aneurysms: A randomised trial. Lancet 2002; 360:1267-74.

5. Raja PV, Huang J, Germanwala AV, Gailloud P, Murphy KP, Tamargo RJ. Microsurgical clipping and endovascular coiling of intracranial aneurysms: A critical review of the literature. Neurosurgery 2008; 62:1187-202; discussion 1202-3.

6. Forsting M, Wanke I. Intracranial vascular malformations and aneurysms: from diagnostic work-up to endovascular therapy. 2nd rev. Edition. Berlin: Springer; 2008.

7. Lim YC, Kim BM, Shin YS, Kim SY, Chung J. Structural limitations of currently available microcatheters and coils for endovascular coiling of very small aneurysms. Neuroradiology 2008; 50:423-7.

8. Nguyen TN, Raymond J, Guilbert F, Roy D, Berube MD, Mahmoud $M$, et al. Association of endovascular therapy of very small ruptured aneurysms with higher rates of procedure-related rupture. J Neurosurg 2008; 108:1088-92.

9. Waseem A, Ahmed I, Hadeel S. Endovascular management of microcerebral aneurysms with diameter smaller than $3 \mathrm{~mm}$ : Is it feasible and safe? Journal of stroke and cerebrovascular diseases: J Stroke Cerebrovasc Dis 2018; 27:1590-8.

10. Figueiredo EG, Teixeira MJ, Spetzler RF, Preul MC. Clinical and surgical experience with the minipterional craniotomy. Neurosurgery 2014; 75:E324-5.

11. Hernesniemi J, Ishii K, Niemelä M, Smrcka M, Kivipelto L, Fujiki $M$, et al. Lateral supraorbital approach as an alternative to the classical pterional approach. Acta Neurochir Suppl 2005; 94:17-21.

12. Esposito G, Dias SF, Burkhardt JK, Fierstra J, Serra C, Bozinov $O$, et al. Selection strategy for optimal keyhole approaches for middle cerebral artery aneurysms: Lateral supraorbital versus minipterional craniotomy. World Neurosurg 2019; 122:e349e357.

13. Salma A, Alkandari A, Sammet S, Ammirati M. Lateral supraorbital approach vs pterional approach: An anatomic qualitative and quantitative evaluation. Neurosurgery 2011; 68:364-72.

14. Weir B, Disney L, Karrison T. Sizes of ruptured and unruptured aneurysms in relation to their sites and the ages of patients. J Neurosurg 2002; 96:64-70.

15. Anokwute MC, Braca JA, Bohnstedt B, DeNardo A, Scott J, Cohen-Gadol $A$, et al. Endovascular treatment of ruptured tiny $(\leqslant 3 \mathrm{~mm})$ intracranial aneurysms in the setting of subarachnoid hemorrhage: A case series of 20 patients and literature review. J Clin Neurosci 2017; 40:52-6.

16. Wu P, Ocak PE, Wang D, Ocak U, Xu S, Li Y, et al. Endovascular treatment of ruptured tiny intracranial aneurysms with low-profile visualized intraluminal support device. J Stroke Cerebrovasc Dis 2019; 28:330-7.

17. Yasargil MG, Fox JL. The microsurgical approach to intracranial aneurysms. Surg Neurol 1975; 3:7-14.

18. Kocaman U, Dalbasti T, Ozer MH, Yilmaz H, Yilmaz MB, Atci IB, et al. The modified lateral supraorbital approach. $\mathrm{Br} J$ Neurosurg 2018; 32:418-3.

19. Cha KC, Hong SC, Kim JS. Comparison between lateral supraorbital approach and pterional approach in the surgical treatment of unruptured intracranial aneurysms. J Korean Neurosurg Soc 2012; 51:334-7.

20. Madhugiri VS, Ambekar S, Pandey P, Guthikonda B, Bollam P, Brown $B$, et al. The pterional and suprabrow approaches for aneurysm surgery: A systematic review of intraoperative rupture rates in 9488 aneurysms. World Neurosurg 2013; 80:836-44. 